\title{
Lymphotoxin-alpha polymorphisms and presence of cancer in I,536 consecutive autopsy cases
}

\author{
Kako Takei ${ }^{1}$, Shinobu Ikeda1, Tomio Arai ${ }^{2}$, Noriko Tanaka ${ }^{3}$, \\ Masaaki Muramatsu*1 and Motoji Sawabe ${ }^{2}$
}

Address: ${ }^{1}$ Department of Molecular Epidemiology, Medical Research Institute, Tokyo Medical and Dental University, Tokyo, 101-0062, Japan, ${ }^{2}$ Department of Pathology, Tokyo Metropolitan Geriatric Hospital, Tokyo, 173-0015, Japan and ${ }^{3}$ Department of Biostatistics, Harvard School of Public Health, Boston, MA, 02115, USA

Email: Kako Takei - takei@comilgr.com; Shinobu Ikeda - sikeda.epi@mri.tmd.ac.jp; Tomio Arai - arai@tmig.or.jp;

Noriko Tanaka - nytanaka@m.u-tokyo.ac.jp; Masaaki Muramatsu* - muramatsu.epi@mri.tmd.ac.jp; Motoji Sawabe - sawabe@tmig.or.jp

* Corresponding author

Published: 13 August 2008

BMC Cancer 2008, 8:235 doi:10.1 186//47|-2407-8-235

This article is available from: http://www.biomedcentral.com//47/-2407/8/235

(c) 2008 Takei et al; licensee BioMed Central Ltd.

This is an Open Access article distributed under the terms of the Creative Commons Attribution License (http://creativecommons.org/licenses/by/2.0), which permits unrestricted use, distribution, and reproduction in any medium, provided the original work is properly cited.
Received: 20 December 2007

Accepted: I3 August 2008

\begin{abstract}
Background: Lymphotoxin-alpha (LTA) is a pro-inflammatory cytokine with anti-tumor activity. The objective of this study was to determine whether LTA polymorphisms influence the presence of cancer.

Methods: LTA polymorphisms C804A (rs104198I, T60N) and T495C (rs2229094, CI3R) were determined in 1,536 consecutive autopsy cases and were registered in the Japanese singlenucleotide polymorphisms (SNPs) for geriatric research (JG-SNP) Internet database. Tumors were systematically reviewed, pathologically confirmed, and assessed in relation to LTA genotype.

Results: The study population consisted of 827 males and 709 females, with a mean age of 80 years. Altogether, we studied 606 subjects without cancer and 930 subjects with cancer of the stomach $(n=183)$, lung $(n=164)$, colon or rectum $(n=143)$, or other sites. The presence of cancer was higher in males than in females. The C804A and T495C polymorphisms were associated with cancer in males $(C A+A A: C C$, adjusted $\mathrm{OR}=0.72,95 \% \mathrm{Cl}=0.53-0.99 ; \mathrm{TC}+\mathrm{CC}$ : TT, adjusted $\mathrm{OR}=1.45,95 \% \mathrm{Cl}=1.04-2.02$; respectively) but not in females. In males, the C804A polymorphism was associated with lung cancer $(C A+A A: C C$, adjusted $O R=0.60,95 \% \mathrm{Cl}=0.37$ - 0.97), whereas the T495C polymorphism was associated with gastric cancer (TC + CC: TT, adjusted $\mathrm{OR}=1.68,95 \% \mathrm{Cl}=1.06-2.65)$.
\end{abstract}

Conclusion: We found some evidence of an association between LTA polymorphisms and cancer risk in elderly Japanese men. Further studies in larger populations should examine this hypothesis.

\section{Background}

Elderly people often experience chronic and low-grade inflammation, whereupon the levels of certain inflammatory serum markers (e.g., C-reactive protein) and proinflammatory cytokines (e.g., tumor necrosis factor-alpha and interleukin-6) are elevated. However, increases in circulating inflammatory markers are often not noticeable in healthy elderly persons and are much below the levels experienced during acute infections [1,2]. Moreover, it is well known that the prevalence of cancer increases sharply 
with age, and that the majority of cancer cases occur in patients over the age of 65 [3]. Persistent inflammation is thought to trigger certain cancers, particularly those of the stomach, colon, and lung [4]. Although it has been established that the risks of cancer and inflammation increase with age [2], the influence of inflammatory cytokine polymorphisms on the genetic predisposition to cancer remains unclear.

Lymphotoxin-alpha (LTA), a member of the tumor necrosis factor (TNF) family of cytokines, was initially isolated on the basis of an anti-tumor activity. Later, this cytokine was shown to have inflammatory and immunologic activities [5]. The LTA gene is located within the class III region of the major histocompatibility complex (MHC) in chromosome 6p21.3 [6]. LTA plays a key role in communication between lymphocytes and stromal cells, thereby eliciting cytotoxic effects on cancer cells $[7,8]$. LTA induces the expression of vascular cell-adhesion molecule 1 (VCAM1) on vascular endothelial cells and recruits natural killer (NK) cells to parenchymal organs and tumor lesions [9]. NK cells have nonspecific host-defense mechanisms that aid in tumor rejection and protection from metastases. Previous studies have shown that tumor growth and metastasis are enhanced in LTA-deficient mice, which produce NK cells that have reduced antitumor potential $[10,11]$. Thus, LTA signaling plays an important role in anti-tumor surveillance via the maturation and recruitment of NK cells.

Previous studies have examined the relationship between LTA polymorphisms and various cancers, and found that the NcoI restriction fragment length polymorphism (A252G) in the first intron is in tight linkage disequilibrium with $\mathrm{C} 804 \mathrm{~A}$, resulting in the substitution of threonine with asparagine at codon 60 in exon $3[12,13]$. The LTA 252G allele increases LTA at the levels of mRNA and protein [12]. Previous studies have found that the relationship between the risk of cancer and the rate of survival among those with the LTA polymorphism varies according to the cancer type [14-17]. However, the effect of LTA polymorphisms on the presence of cancer in general is not known.

Toward this end, we searched for two non-synonymous polymorphisms, C804A (rs1041981, T60N) and T495C (rs2229094, C13R), in consecutive autopsy cases and determined whether they influenced cancer presence.

\section{Methods \\ Subjects}

The study population consisted of 1,536 consecutive autopsy cases performed at the Tokyo Metropolitan Geriatric Hospital between 1995 and 2004. LTA polymorphisms were registered in the Internet database of
Japanese single-nucleotide polymorphisms (SNPs) for geriatric research (JG-SNP) [18]. Subjects were enrolled regardless of the cause of death and autopsies were performed on $40 \%$ of patients who died at the hospital. The study population included subjects who died from malignant disease $(33 \%)$, coronary heart disease $(20 \%)$, and pneumonia (13\%). These proportions were similar to the causes of death reported in a survey conducted by the Ministry of Health, Labor and Welfare of Japan http:// www.mhlw.go.jp/toukei/saikin/hw/jinkou/tokusyu/ gaikoku07/09d.html which found that $30 \%$ of the Japanese population died from malignant disease, $16 \%$ from coronary heart disease, and 10\% from pneumonia. The presence, absence, and histopathology of cancers were determined from autopsy findings. Histories of smoking and alcohol drinking status were retrospectively determined from medical records, and subjects were classified as smokers (including ex-smokers) versus non-smokers, and alcohol drinkers versus non-drinkers. Information on smoking and alcohol drinking status was missing from 109 and 118 patient histories, respectively. Written informed consent was obtained from the family of each patient at the time of autopsy. The study protocol was approved by the ethical Committees of the Tokyo Metropolitan Geriatric Hospital and the Tokyo Medical and Dental University.

\section{Genotyping}

Genomic DNA was extracted from the renal cortex using a standard procedure. LTA polymorphisms were genotyped via melting curve analysis [19]. PCR primers and probes were as follows. For LTA C804A (rs1041981, T60N): 5'GTT GGC CTC ACA CCT TCA-3' (forward primer), 5'-TGG ATG CTT GGG TTC CTG-3' (reverse primer), CAG CAC CCT CAA ACC TGC-Fluorescein (anchor probe), and LC Red 640-GCT CAC CTC ATT GGT AAA CAT CCA CCT GAC CTC C-Phosphate (detection probe). For LTA T495C (rs2229094, C13R): 5'-CTC TTT CTC TGC AGG TTC TC-3' (forward primer), 5'-GCT CTA GGG CTC AAG GTT T-3' (reverse primer), CTC CCA AGG GTG GGT G-Fluorescein (anchor probe), and LCRed640-CAC CAC CCT ACA CCT CCT CCT TCT GG-Phosphate (detection probe). The PCR amplification reaction was performed in $5 \mu$ l volume using the LightCycler PCR kit (Roche Diagnostics, Penzberg, Germany). Each well contained $1 \times$ PCR buffer, 4 $\mathrm{mM}$ of $\mathrm{MgCl} 2,0.2 \mathrm{mM}$ of dNTPs, $0.05 \mu \mathrm{M}$ of forward primer, $0.5 \mu \mathrm{M}$ of reverse primer, $0.2 \mu \mathrm{M}$ of each anchor and detection probe, 0.1 U of Faststart Taq polymerase, and $10 \mathrm{ng}$ of genomic DNA. The cycling protocol was performed as follows: initial denaturation at $94^{\circ} \mathrm{C}$ for 10 min; followed by 40 cycles of $94^{\circ} \mathrm{C}$ for $15 \mathrm{sec}, 55^{\circ} \mathrm{C}$ for $15 \mathrm{sec}$, and $72^{\circ} \mathrm{C}$ for $15 \mathrm{sec}$; and a final extension step at $72^{\circ} \mathrm{C}$ for $10 \mathrm{~min}$. After completion of PCR, the plates were heated from $40^{\circ} \mathrm{C}$ to $90^{\circ} \mathrm{C}$ with a gradient of $0.1^{\circ} \mathrm{C}$ per second immediately before melting temperature analysis 
on the LightCycler 480 Instrument (Roche Diagnostics). The genotype was determined based on melting profiles automatically classified by LightCycler Genotyping software (Roche Diagnostics). C804A and T495C polymorphisms were genotyped with success rates of $96 \%$ and $95 \%$, respectively. Genotyping accuracy was confirmed by sequencing randomly selected samples. The melting temperature analysis correlated entirely with the direct sequencing results.

\section{Statistical analysis}

Statistical analysis was performed using SAS software, version 9.1.3 (SAS Institute Inc., Cary, NC). The distribution of subjects with and without cancer, and associations with other variables, were compared using Fisher's exact test. Hardy-Weinberg equilibrium (HWE) was assessed using a permutation test. Odds ratio (OR) and 95\% confidence interval (CI) were calculated using an unconditional logistic regression model that compared genotype frequencies in subjects with and without cancer, with respect to LTA polymorphisms. The OR was adjusted by age, sex, smoking, and alcohol drinking status. This study had a statistical power of 0.8 to detect an OR of 1.5 in carriers of at least one polymorphic allele, compared with carriers of homozygous wild-type alleles. LTA haplotype frequencies were estimated using Haploview software http:// www.broad.mit.edu/mpg/haploview/. Chi-square values were calculated to determine the distribution of LTA haplotypes among subjects with and without cancer. A probability level of 5\% was considered statistically significant for all analyses.

\section{Results}

Characteristics of the study subjects

Selected demographic variables, including the sites and histological types of cancers, and risk factors are shown in Table 1 (Additional file 1). The mean age of subjects was $80.2 \pm 8.9$ years. The study population consisted of 827 (54\%) males and 709 (46\%) females. Altogether, we studied 606 subjects without cancer and 930 subjects with cancer. When the total number of subjects was divided by the number of subjects over or under the median age of 80 years, the distributions of subjects with or without cancer did not significantly differ. The frequency of cancer was significantly higher in males than in females. No differences in smoking and alcohol drinking status between cancer-bearing and cancer-free subjects were observed. The most frequent sites of cancer were the stomach $(\mathrm{n}=$ $183)$, lung $(\mathrm{n}=164)$, and colon or rectum $(\mathrm{n}=143)$.

\section{Genotype and allele frequencies of LTA polymorphisms} C804A and T495C were genotyped in all subjects. The genotype frequencies of C804A were $37 \%$ for CC, $48 \%$ for $\mathrm{CA}$, and $15 \%$ for $\mathrm{AA}$. The minor allele frequency was $39 \%$, and the allele distribution was consistent with $\operatorname{HWE}(\mathrm{p}=$
0.65). The genotype frequencies of $\mathrm{T} 495 \mathrm{C}$ were $66 \%$ for $\mathrm{TT}, 31 \%$ for TC, and 3\% for CC. The minor allele frequency was $19 \%$ and the allele distribution was consistent with HWE $(\mathrm{p}=0.48)$.

\section{Association of LTA polymorphisms with cancer overall}

The associations between C804A and T495C polymorphisms and the presence of cancer are shown in Table 2 (Additional file 1). Among cancer-free subjects, the frequencies of the $\mathrm{C} 804 \mathrm{~A}$ genotypes $\mathrm{CC}, \mathrm{CA}$, and $\mathrm{AA}$ were $33 \%, 49 \%$, and $18 \%$, respectively, in males, and 33\%, $54 \%$, and $13 \%$, respectively, in females (Table 3 ) (Additional file 1). In comparison with the CC genotype, the CA genotype was associated with a significantly lower presence of cancer in all subjects (adjusted OR $=0.78,95 \% \mathrm{CI}$ $=0.61-0.99)$. In males, the CA + AA genotype was associated with a significantly lower presence of cancer compared with the CC genotype (adjusted OR $=0.72,95 \% \mathrm{CI}$ $=0.53-0.99)$. The association between the C804A polymorphism and cancer in females was not significant (CA + AA: CC, adjusted OR $=0.92,95 \%$ CI $=0.66-1.29$ ) . Additionally, cancer frequency was not associated with C804A (data not shown).

In cancer-free subjects, the frequency of the T495C genotypes TT, TC, and CC were $70 \%, 28 \%$, and $2 \%$, respectively, in males, and $66 \%, 31 \%$, and $3 \%$, respectively, in females (Table 3 in Additional file 1). Compared with the TT genotype, the CC genotype was associated with a significantly higher presence of cancer (adjusted $\mathrm{OR}=2.24$, $95 \% \mathrm{CI}=1.09-4.61)$. In males, the TC + CC genotype was associated with a significantly higher presence of cancer, compared with the TT genotype (adjusted OR $=1.45$, $95 \% \mathrm{CI}=1.04-2.02)$. The association between the T495C polymorphism and cancer in females was not significant (TC + CC: $\mathrm{TT}$, adjusted OR $=1.08,95 \% \mathrm{CI}=0.77$ $-1.50)$.

Additionally, we explored the relationship between the presence of $\mathrm{C} 804 \mathrm{~A}$ and $\mathrm{T} 495 \mathrm{C}$ haplotypes and tumor formation. C804A and T495C appeared to be present in moderate linkage disequilibrium $\left(\mathrm{r}^{2}=0.15, \mathrm{D}^{\prime}=1.0\right)$ and three major haplotypes were identified (495T-804C, 495T-804A, and 495C-804C). None of these haplotypes were associated with the presence of cancer (data not shown).

\section{Association of LTA polymorphisms with specific types of cancers}

Significant associations between LTA polymorphisms and the presence of cancer in males prompted us to explore the relationships between these polymorphisms and the sites of cancer. After analyzing subjects with stomach, lung, colorectal, prostate, breast, liver, biliary tract, kidney or urinary tract, and hematopoietic malignancies, we 
observed a positive association between LTA polymorphisms and lung and stomach cancers (Table 4 in Additional file 1). Among male subjects with lung cancer, the C804A CA + AA genotype was associated with a significantly lower presence of cancer than was the CC genotype (adjusted OR $=0.60,95 \% \mathrm{CI}=0.37-0.97$ ). The association between C804A polymorphism and lung cancer in females was not significant $(\mathrm{CA}+\mathrm{AA}$ : CC, adjusted OR = $0.65,95 \% \mathrm{CI}=0.35-1.23)$.

The T495C polymorphism was not associated with the presence of lung cancer, but the TC + CC genotype was associated with a significantly higher presence of gastric cancer in males, compared with the TT genotype (adjusted $\mathrm{OR}=1.68,95 \% \mathrm{CI}=1.06-2.65)$. The association between T495C polymorphism and gastric cancer was not significant in females (TC + CC: TT, adjusted OR $=1.16,95 \%$ CI $=0.61-2.21)$.

\section{Discussion}

Our findings demonstrate that the LTA polymorphism $\mathrm{C} 804 \mathrm{~A}$ is associated with a lower presence of cancer, particularly lung cancer, in elderly Japanese men, consistent with previous studies showing that LTA has anti-tumor activity.

Several laboratories have recently published a functional analysis of the LTA C804A (T60N). Compared with the C allele, the A allele is more bioactive with regards to the induction of VCAM1 in cultured human coronary-artery smooth muscle cells [13]. Furthermore, C804A is present in very high linkage disequilibrium with another polymorphism, LTA A252G. These two polymorphisms have been shown to be completely concordant in Japanese subjects $[16,17]$. Compared with the A allele, the $G$ allele of the A252G genotype confers higher transcriptional activity in Jurkat cells [13] and phytohemagglutinin-stimulated peripheral blood mononuclear cells [12]. Thus, the $\mathrm{C}$ and $\mathrm{A}$ alleles are considered low and high bioactive alleles in LTA, respectively. Accordingly, the data suggest that the lower presence of cancer in our study population may reflect enhanced LTA activity because of the presence of high-bioactive alleles.

The C804A and A252G polymorphisms have been studied with regards to cancer survival rates and risks of developing various type of cancer, including lung [14], stomach $[15,20,21]$, colorectal [22], breast [23], cervix [16], endometrium [17] and bladder [24] cancers, as well as leukemia [25], lymphoma [26,27], and myeloma [28]. The high-bioactive genotype was associated with the risk of developing cancers of the lung [14], colon or rectum [22], non-Hodgkin lymphoma $[26,27]$, and myeloma [28]. Low-bioactive genotypes were associated with the risk of developing cervical [16] and endometrial cancers
[17], but not gastric [15,20,21], breast [23], bladder cancers [24], or leukemia [25]. These differences may be partially explained by the multi-functionality of LTA. LTA can promote cell growth and adhesion, and can potentially favor the growth of certain tumors.

It remains unclear why $\mathrm{C} 804 \mathrm{~A}$ is associated with cancer in males but not in females. However, gender is known to play an important role in the development of various cancers. Recent studies also suggest that cytokine secretion and innate immunity differ between the genders $[29,30]$.

The C13R (T495C) polymorphism has not been as thoroughly studied as the T60N (C804A) polymorphism. Previous study reported there was not an association between the T495C polymorphism and lung cancer [31]. The T495C polymorphism is a haplotype component associated with altered LTA expression and increased levels of vascular- and autoimmune-mediated inflammation [32]. These findings suggest that the T495C polymorphism is also associated with cancer. We observed an association between the $\mathrm{C}$ allele of the T495C polymorphism and the presence of cancer, particularly gastric cancer. However, as the minor allele frequency for this polymorphism was low, these results must be confirmed in a larger study population.

The LTA gene is located within the class III region of the MHC, in the 6p21.3 chromosome [6]. This region contains many other genes, including that encoding the proinflammatory cytokine, TNF. It is well-known that this region shows high degrees of linkage disequilibrium. Thus, associations identified in this study may reflect the effects of other gene variants in this region.

As this was a hospital-based autopsy study, we had limited access to information on lifestyle variables that could potentially influence the development of cancer. Furthermore, although autopsies were performed on many of the deaths (40\%) that occurred in the hospital, and the causes of death among our autopsy cases were similar to those reported in a national survey, we cannot rule out the possibility of selection bias. Such bias can arise from chance of admission, consent to autopsy, cause of death, and autopsy practice. Survival bias is also a possibility, as particular genotypes may be associated with other diseases or influence the lifespan of certain subjects, thereby introducing bias into the study population. However, the C804A genotype frequency was similar to that reported in other Japanese population studies $[16,17]$, and any effects of such possible bias did not cause our results to deviate from HWE. 


\section{Conclusion}

We found some evidence of an association between LTA polymorphisms and cancer risk in elderly Japanese men. Further studies in larger populations should examine this hypothesis.

\section{Competing interests}

The authors declare that they have no competing interests.

\section{Authors' contributions}

KT performed the statistical analysis and drafted the manuscript. SI participated in the design and performed the statistical analysis. TA performed the pathological analysis. NT assessed the data integrity. MM coordinated the study and helped draft the manuscript. MS performed the pathological analysis and helped coordinate the study. All authors read and approved the final manuscript.

\section{Additional material}

\section{Additional file 1}

Tables 1-4. Table 1 - Distribution of selected demographic variables and risk factors. Table 2 - Associations between LTA polymorphisms and overall cancer. Table 3 -Distribution of LTA genotypes in cancer-free and cancer-bearing subjects, lung and gastric cancers. Table 4 - Associations of LTA polymorphisms with lung cancer and gastric cancer.

Click here for file

[http://www.biomedcentral.com/content/supplementary/14712407-8-235-S1.doc]

\section{Acknowledgements}

This work was partly supported by grant-in-aid from the Ministry of Health, Labor and Welfare, and the Ministry of Education, Culture, Sports, Science and Technology, Japan.

We thank Kenta Takahashi, of the Tokyo Medical and Dental University, for assisting genotype analysis.

\section{References}

I. Bruunsgaard H, Pedersen M, Pedersen BK: Aging and proinflammatory cytokines. Current opinion in hematology 200I, 8(3): $|3|-\mid 36$.

2. Caruso C, Lio D, Cavallone L, Franceschi C: Aging, longevity, inflammation, and cancer. Annals of the New York Academy of Sciences 2004, 1028: I-13.

3. Serrano M, Blasco MA: Cancer and ageing: convergent and divergent mechanisms. Nature reviews 2007, 8(9):715-722.

4. Coussens LM, Werb Z: Inflammation and cancer. Nature 2002, 420(69|7):860-867.

5. Aggarwal BB: Signalling pathways of the TNF superfamily: a double-edged sword. Nature reviews 2003, 3(9):745-756.

6. Carroll MC, Katzman P, Alicot EM, Koller BH, Geraghty DE, Orr HT, Strominger JL, Spies T: Linkage map of the human major histocompatibility complex including the tumor necrosis factor genes. Proceedings of the National Academy of Sciences of the United States of America 1987, 84(23):8535-8539.

7. Vassalli P: The pathophysiology of tumor necrosis factors. Annual review of immunology 1992, 10:4 I -452.

8. Ware CF, Crowe PD, Grayson MH, Androlewicz MJ, Browning JL: Expression of surface lymphotoxin and tumor necrosis fac- tor on activated T, B, and natural killer cells. J Immunol 1992, I 49( I 2):388I-3888.

9. Fogler WE, Volker K, McCormick KL, Watanabe M, Ortaldo JR, Wiltrout RH: NK cell infiltration into lung, liver, and subcutaneous B 16 melanoma is mediated by VCAM-I/VLA-4 interaction. J Immunol 1996, I 56( I 2):4707-47|4.

10. Ito D, Back TC, Shakhov AN, Wiltrout RH, Nedospasov SA: Mice with a targeted mutation in lymphotoxin-alpha exhibit enhanced tumor growth and metastasis: impaired NK cell development and recruitment. I Immunol 1999, 163(5):2809-2815.

II. Smyth MJ, Johnstone RW, Cretney E, Haynes NM, Sedgwick JD, Korner H, Poulton LD, Baxter AG: Multiple deficiencies underlie NK cell inactivity in lymphotoxin-alpha gene-targeted mice. J Immunol 1999, I63(3): 1350-1353.

12. Messer G, Spengler U, Jung MC, Honold G, Blomer K, Pape GR, Riethmuller G, Weiss EH: Polymorphic structure of the tumor necrosis factor (TNF) locus: an Ncol polymorphism in the first intron of the human TNF-beta gene correlates with a variant amino acid in position 26 and a reduced level of TNFbeta production. The Journal of experimental medicine 1991, 173(1):209-219.

13. Ozaki K, Ohnishi Y, lida A, Sekine A, Yamada R, Tsunoda T, Sato $H$, Sato $\mathrm{H}$, Hori M, Nakamura $Y$, Tanaka T: Functional SNPs in the lymphotoxin-alpha gene that are associated with susceptibility to myocardial infarction. Nature genetics 2002, 32(4):650-654.

14. Shimura T, Hagihara M, Takebe K, Munkhbat B, Odaka T, Kato H, Nagamachi $Y$, Tsuji K: The study of tumor necrosis factor beta gene polymorphism in lung cancer patients. Cancer 1994, 73(4): $1184-1188$.

15. Shimura T, Hagihara M, Takebe K, Munkhbat B, Ogoshi K, Mitomi T, Nagamachi $Y$, Tsuji K: 10.5-kb homozygote of tumor necrosis factor-beta gene is associated with a better prognosis in gastric cancer patients. Cancer 1995, 75(6 Suppl): | 450- 1453.

16. Niwa Y, Hirose K, Matsuo K, Tajima K, Ikoma Y, Nakanishi T, Nawa A, Kuzuya K, Tamakoshi A, Hamajima N: Lymphotoxin-alpha polymorphism and the risk of cervical cancer in Japanese subjects. Cancer letters 2005, 21 8(I):63-68.

17. Niwa Y, Ito H, Matsuo K, Hirose K, Ito N, Mizuno M, Hamajima N, Tajima K, Nakanishi T: Lymphotoxin-alpha polymorphisms and the risk of endometrial cancer in Japanese subjects. Gynecologic oncology 2007, 104(3):586-590.

18. Sawabe M, Arai T, Kasahara I, Esaki Y, Nakahara K, Hosoi T, Orimo $\mathrm{H}$, Takubo K, Murayama S, Tanaka N: Developments of geriatric autopsy database and Internet-based database of Japanese single nucleotide polymorphisms for geriatric research (JGSNP). Mechanisms of ageing and development 2004, I 25(8):547-552.

19. Oda K, Tanaka N, Arai T, Araki J, Song Y, Zhang L, Kuchiba A, Hosoi T, Shirasawa T, Muramatsu M, Sawabe M: Polymorphisms in proand anti-inflammatory cytokine genes and susceptibility to atherosclerosis: a pathological study of 1503 consecutive autopsy cases. Human molecular genetics 2007, I 6(6):592-599.

20. Guo W, Wang N, Li $Y$, Zhang JH: Polymorphisms in tumor necrosis factor genes and susceptibility to esophageal squamous cell carcinoma and gastric cardiac adenocarcinoma in a population of high incidence region of North China. Chinese medical journal 2005, I | 8(22): 1870-1878.

2I. Lee SG, Kim B, Yook JH, Oh ST, Lee I, Song K: TNF/LTA polymorphisms and risk for gastric cancer/duodenal ulcer in the Korean population. Cytokine 2004, 28(2):75-82.

22. de Jong MM, Nolte IM, te Meerman GJ, van der Graaf WT, de Vries EG, Sijmons RH, Hofstra RM, Kleibeuker JH: Low-penetrance genes and their involvement in colorectal cancer susceptibility. Cancer Epidemiol Biomarkers Prev 2002, I I (I I): I332-I 352.

23. Gaudet MM, Egan KM, Lissowska J, Newcomb PA, Brinton LA, TitusErnstoff L, Yeager M, Chanock S, Welch R, Peplonska B, TrenthamDietz A, Garcia-Closas M: Genetic variation in tumor necrosis factor and lymphotoxin-alpha (TNF-LTA) and breast cancer risk. Human genetics 2007, I 2 I(3-4):483-490.

24. Nonomura N, Tokizane T, Nakayama M, Inoue H, Nishimura K, Muramatsu M, Okuyama A: Possible correlation between polymorphism in the tumor necrosis factor-beta gene and the clinicopathological features of bladder cancer in Japanese patients. Int J Urol 2006, I3(7):97| -976. 
25. Demeter J, Porzsolt F, Ramisch S, Schmidt D, Schmid M, Messer G: Polymorphism of the tumour necrosis factor-alpha and lymphotoxin-alpha genes in chronic lymphocytic leukaemia. British journal of haematology 1997, 97(I): 107-1 I2.

26. Purdue MP, Lan Q, Kricker A, Grulich AE, Vajdic CM, Turner J, Whitby D, Chanock S, Rothman N, Armstrong BK: Polymorphisms in immune function genes and risk of non-Hodgkin lymphoma: findings from the New South Wales non-Hodgkin Lymphoma Study. Carcinogenesis 2007, 28(3):704-7II.

27. Wang SS, Cerhan JR, Hartge P, Davis S, Cozen W, Severson RK, Chatterjee N, Yeager M, Chanock SJ, Rothman N: Common genetic variants in proinflammatory and other immunoregulatory genes and risk for non-Hodgkin lymphoma. Cancer research 2006, 66(19):977।-9780.

28. Davies FE, Rollinson S], Rawstron AC, Roman E, Richards S, Drayson M, Child JA, Morgan GJ: High-producer haplotypes of tumor necrosis factor alpha and lymphotoxin alpha are associated with an increased risk of myeloma and have an improved progression-free survival after treatment. J Clin Oncol 2000, 18(15):2843-285I.

29. Moxley G, Posthuma D, Carlson P, Estrada E, Han J, Benson LL, Neale MC: Sexual dimorphism in innate immunity. Arthritis and rheumatism 2002, 46(I):250-258.

30. Aulock SV, Deininger S, Draing C, Gueinzius K, Dehus O, Hermann $C:$ Gender difference in cytokine secretion on immune stimulation with LPS and LTA. J Interferon Cytokine Res 2006, 26(I 2):887-892

31. Engels EA, Wu X, Gu J, Dong Q, Liu J, Spitz MR: Systematic evaluation of genetic variants in the inflammation pathway and risk of lung cancer. Cancer research 2007, 67(13):6520-6527.

32. Belfer I, Buzas B, Hipp H, Dean M, Evans C, Lorincz I, Max MB, Goldman $D$ : Haplotype structure of inflammatory cytokines genes (ILIB, IL6 and TNF/LTA) in US Caucasians and African Americans. Genes and immunity 2004, 5(6):505-5I2.

\section{Pre-publication history}

The pre-publication history for this paper can be accessed here:

http://www.biomedcentral.com/1471-2407/8/235/pre

pub

Publish with Bio Med Central and every scientist can read your work free of charge

"BioMed Central will be the most significant development for disseminating the results of biomedical research in our lifetime. "

Sir Paul Nurse, Cancer Research UK

Your research papers will be:

- available free of charge to the entire biomedical community

- peer reviewed and published immediately upon acceptance

- cited in PubMed and archived on PubMed Central

- yours - you keep the copyright
BioMedcentral 